\title{
Enquête
}

Archives de la revue Enquête

2 | 1995

Usages de la tradition

\section{Les traditions sociologiques}

Sociological traditions

\section{Randall Collins}

Traducteur : Jean-Louis Fabiani

\section{(2) OpenEdition \\ Journals}

Édition électronique

URL : http://journals.openedition.org/enquete/302

DOI : 10.4000/enquete.302

ISSN : 1953-809X

Éditeur :

Cercom, Éditions Parenthèses

Édition imprimée

Date de publication : 31 décembre 1995

Pagination : 11-38

\section{Référence électronique}

Randall Collins, "Les traditions sociologiques », Enquête [En ligne], 2 | 1995, mis en ligne le 10 juillet

2013, consulté le 12 novembre 2019. URL : http://journals.openedition.org/enquete/302 ; DOI :

$10.4000 /$ enquete.302

Ce document a été généré automatiquement le 12 novembre 2019. 


\title{
Les traditions sociologiques
}

\author{
Sociological traditions
}

\section{Randall Collins}

Traduction : Jean-Louis Fabiani

1 Une tradition, c'est à la fois ce dont on se souvient et ce avec quoi on s'identifie. Le fondement de toute tradition est une communauté, un être collectif au sens durkheimien. Une telle communauté peut prendre la forme d'un véritable réseau intergénérationnel de transmission, ou être purement imaginaire, comme les pseudo-lignages dont Weber avait remarqué l'existence au sein de certaines coalitions tribales qui s'étaient inventé des ancêtres pour les besoins de leur propre organisation politique. Dans les deux cas, la communauté existante met en forme sa tradition, principalement en fonction de la relation qu'elle développe avec les représentations symboliques rivales des collectivités antagonistes.

2 Les sociologues s'enorgueillissent d'être les iconoclastes qui arrachent les voiles des traditions. Leurs propres réseaux intellectuels sont pourtant organisés par des traditions. Ceci revient simplement à dire que le monde intellectuel a sa propre organisation sociale, même si les intellectuels peuvent s'opposer aux traditionalistes tels qu'ils existent dans la vie politique et sociale ordinaire. L'identité professionnelle des sociologues a commencé à se constituer en prenant la tradition comme objet, à travers le thème du contraste entre tradition et modernité dans les diverses formulations qu'en ont données Saint-Simon et Comte, Tönnies et Weber. Les théoriciens ont considéré que la tradition offrait plus de ressources pour l'analyse qu'un simple construit historique, en en faisant un élément constitutif de l'ordre social en général : nous pouvons le constater à travers la primauté que Durkheim confère à la solidarité mécanique sur la solidarité organique, le système de valeurs de Parsons, les structures de Lévi-Strauss ou l'habitus de Bourdieu. Dans un lignage différent, on peut citer le stock de savoir commun pris comme allant de soi selon Schutz et l'ethnométhodologie de Garfinkel. Les sociologues reconnaissent bien sûr que des formes opposées aux types traditionnels d'action et de croyance existent aussi : mais même le conflit a ses traditions et les mouvements sociaux les plus iconoclastes acquièrent leurs propres schèmes d'organisation collective et partant leurs propres traditions. 
Chez les sociologues particulièrement (peut-être chez les intellectuels en général), nous trouvons trois types de traditions, plus un quatrième, l'anti-tradition. Ce n'est pas leur contenu (comme le conflit, ou la tradition de l'analyse durkheimienne, etc.) qui constitue ici le principe de division, mais les relations sociales qui se développent entre ces collectivités intellectuelles et leur propre symbolisme traditionnel.

\section{Les traditions loyalistes ou orientées par des héros}

On désigne ainsi les traditions centrées autour d'un fondateur héroïque, qui commande la loyauté des disciples à travers la succession des générations. Les érudits consacrent leur carrière à faire l'exégèse des textes du fondateur. On peut appliquer des idées classiques à des problèmes et des matériaux nouveaux, mais toujours avec le souci de montrer que la théorie conserve son pouvoir explicatif et qu'elle continue de bien se défendre contre les positions rivales dans le monde intellectuel contemporain. Les débats sur l'hérésie constituent une forme majeure d'action intellectuelle au sein des communautés loyalistes : les factions se disputent pour savoir laquelle maintient le mieux l'esprit ou la lettre de l'original, et laquelle, par son déviationnisme, perd la qualité de membre d'un lignage. Un tel loyalisme centré autour du fondateur nous fait penser à l'histoire des religions, aux disciples et aux gardiens des textes dans les traditions de Jésus, de Mahomet ou de Confucius. En sociologie, le traditionalisme loyaliste inclut quelques-uns des plus grands noms : Marx, Weber, George Herbert Mead, Freud.

5 La force de ces traditions varie au cours du temps. La tradition marxienne est restée pendant plusieurs décennies confinée à l'univers de l'activisme politique; au cours des années soixante et soixante-dix, celle-ci est devenue une faction importante au sein de la sociologie universitaire en Europe et dans le monde anglophone, avant de s'effacer à nouveau. Le loyalisme weberien a vu croître son importance au cours du temps. En Allemagne, Weber n'était qu'un nom parmi d'autres dans les années vingt : ce n'est qu'au début des années soixante, lorsque des chercheurs américains (certains ayant immigré dans les années trente) ont réévalué sa réputation, que son statut s'est élevé au point qu'il est devenu le centre d'une école. Aux États-Unis, dans les années quarante et cinquante, Weber était seulement un ingrédient utilisé comme ressource additionnelle par diverses factions sociologiques (structuro-fonctionnalisme, activisme de Wright Mills, théorie du conflit, théorie des organisations). Dans les années quatre-vingts et quatre-vingt-dix l'érudition weberienne est devenue la province la plus importante dans le domaine de l'analyse des textes classiques. Les disciples de Mead, regroupés sous la bannière de l'interactionnisme symbolique, ont constitué un groupe orienté de façon prédominante par rapport à la recherche de terrain pendant des décennies à partir de leur constitution dans les années trente aux États-Unis; depuis les années soixante-dix, avec la fondation d'une Society for Symbolic Interaction et le développement d'une érudition textuelle à propos de Mead et des autres auteurs classiques du pragmatisme, le mode de tradition centré sur un héros semble avoir gagné ici aussi la prééminence.

On ne doit pas conclure que la créativité intellectuelle ne peut trouver place au sein d'une tradition loyaliste. Ce qu'une faction parmi les interprètes traite d'hérésie peut constituer une innovation conduisant à des conceptions et des recherches nouvelles. Dans la sociologie et l'histoire sociale américaine de la fin des années soixante-dix, l'analyse du " système-monde » développée par Wallerstein a fait l'objet d'une attaque à travers une controverse bien connue, dite de Brenner ${ }^{1}$ : cette notion était assimilée à un «marxisme 
néo-smithien ». Loin de disqualifier l'école du système-monde, cette critique provenant du marxisme orthodoxe a suscité à son égard plus d'attention dans la conjoncture intellectuelle du moment. Des années quarante aux années soixante, les débats sur la signification des textes divers de Weber se sont développés entre les disciples fonctionnalistes de Parsons et leurs rivaux de diverses appartenances sociologiques. Parsons et ses collaborateurs s'étaient servi de Weber pour conforter la position centrale de la notion de système de valeurs dans l'analyse sociologique en commençant par traduire et commenter L'Éthique protestante et l'esprit du capitalisme, puis les définitions conceptuelles abstraites qui constituent la première partie de Wirtschaft und Gesellschaft, et les chapitres constituant la Sociologie de la religion. Hans Gerth et C. Wright Mills, le premier émigré en provenance de l'école de Francfort, le second pragmatiste de gauche, s'associèrent pour la contre-attaque en présentant la traduction anglaise des écrits de Weber sur classe, status et parti, sur la bureaucratie, sur la politique, et d'autres textes sur les fondements multidimensionnels des luttes sociales et de la domination. Ces écrits devinrent un point de ralliement pour les tenants de la théorie du conflit, se situant à michemin entre le marxisme et le fonctionnalisme. C'est au cercle réuni autour de Gerth et de Mills que nous devons les traductions des études de Weber sur les religions chinoise et indienne et sur le judaïsme antique. Celles-ci donnèrent son premier élan à un mouvement de comparatisme historique (représenté par Bendix, Lenski, Moore, Skocpol, Tilly et leurs successeurs) qui a pris généralement une position weberienne de gauche quelquefois évoquée sous l'étiquette de " théorie du conflit ». Nous constatons à partir de cet exemple que la lutte pour l'appropriation des éléments disparates d'une tradition peut être source d'innovations fécondes dans le processus même de constitution d'une tradition de recherche.

7 Une organisation sociale de la spécialisation intellectuelle très différente peut également prendre forme. Les exemples que constitue l'érudition marxienne et weberienne, si on les considère des années trente aux années quatre-vingt-dix, montrent que le concept de « théorie » sociale peut être approprié par des factions qui l'identifient à l'étude de textes classiques per se. Ce genre de traditionalisme favorise la tendance au divorce entre d'un côté la «théorie» et de l'autre les travaux empiriques ainsi que la découverte de nouveaux principes ou la modification d'anciens principes. Ici le traditionalisme du mode loyaliste est associé avec les slogans méta-théoriques du "postpositivisme» dans des positions comme celle de Jeffrey Alexander ${ }^{2}$ qui soutient que le travail de théorisation est autonome par rapport à l'observation concrète et partant des généralisations empiriques qui peuvent se développer à partir du concret. Cette position est la contrepartie épistémologique de la pratique qui consiste à produire de la théorie à partir de la simple juxtaposition de textes plus anciens: Talcott Parsons a été le pionnier de ce mode de théorisation avec The Structure of Social Action en 1937 (ouvrage dans lequel une grande théorie était construite à partir d'une synthèse entre Durkheim, Weber, Marschall et Pareto). Il a été suivi par Alexander lui-même, dont la production théorique est un métacommentaire sur le panthéon modifié constitué par Marx, Weber et Parsons lui-même. Il a été aussi suivi par Habermas dans la Théorie de l'agir communicationnel (métacommentaire du même panthéon que celui d'Alexander, avec l'addition de Mead), par Giddens et par d'autres. Il s'agit ici d'un mode qu'on pourrait qualifier de « loyalisme de la synthèse ", combinaison apparemment paradoxale des loyalismes, puisqu'on se tient au plus près des principes directeurs de chaque lignage. On peut qualifier autrement ce mode en affirmant qu'il s'agit d'une forme de loyalisme à l'égard de la tradition du type de construction théorique inauguré par Parsons. 
8 La grande faiblesse du fonctionnalisme parsonien tenait au fait qu'il privilégiait l'intégration de son schème conceptuel aux dépens des constats de la recherche empirique. La recherche menée depuis 1960 sur la stratification, les mouvements sociaux, les organisations, la lutte politique, le développement de l'État, l'identité sexuelle, la micro-interaction et d'autres thèmes ne doit pas ses découvertes à l'application d'hypothèses fonctionnalistes. Ces recherches ont au contraire stimulé des points de vue rivaux qui prêtaient plus d'attention à la négociation, au conflit et à la domination. La tradition fonctionnaliste a assuré son salut en s'affranchissant par principe du travail empirique : si le travail théorique est autonome, il ne peut en effet faire l'objet d'aucune disqualification empirique.

9 Nous constatons ici un changement dans la position des théoriciens au sein de ces traditions entre les classiques et leurs répliques contemporaines. Marx et Engels, Weber, Durkheim et même Mead dans son domaine n'ont jamais considéré la théorie comme étant autonome par rapport aux analyses concrètes que leur fournissait l'observation du monde. Les éléments dans lesquels nous reconnaissons des points classiques de leurs théories trouvent bien souvent leur origine dans l'observation attentive du monde. Le travail théorique de Marx et de Weber n'aurait pu voir le jour sans leurs très vastes recherches sur le capitalisme, pas plus que celui de Durkheim et Mauss n'aurait pu se développer sans le programme systématique de comparaison qui a pris corps à travers l' Année sociologique. Les analyses de Mead lui-même n'auraient pas pu voir le jour indépendamment de ses observations sur les enfants et les animaux. Il est banal, mais néanmoins essentiel, d'affirmer que l'innovation en sciences sociales est le résultat de l'influence réciproque de la théorie et de la recherche empirique. Les grands découvreurs lisent à la fois les livres de leurs prédécesseurs et le livre du monde. Aujourd'hui le sociologue traditionaliste ne lit que les écrits des théoriciens. Le développement de l'orientation loyaliste au sein de ces traditions à la fin $\mathrm{du} \mathrm{xx}^{\mathrm{e}}$ siècle est l'indicateur d'une séparation croissante des rôles: le spécialiste ès théorie devient le spécialiste ès textes, qui n'a pas besoin de contact avec les chercheurs empiriques. Ce constat paradoxal revient peut-être simplement à dire que les fondateurs de tradition n'étaient pas euxmêmes des traditionalistes.

10 Nous ne devons pas en conclure pour autant que la main morte du passé ne nous lâchera plus désormais. Le développement peut survenir dans l'univers des spécialistes de la pure théorie, même lorsqu'on accorde la préséance à la loyauté à l'intérieur d'une tradition, à la célébration du fondateur comme un héros et à la primauté des textes classiques. L'idéologie de la vie intellectuelle qui prévaut en Chine depuis la dynastie des Han repose sur le fait que le savoir est tout entier contenu dans les écrits classiques du canon confucéen. À certaines périodes dans l'Inde et la Chine médiévales, on a conféré cette position privilégiée aux sûtra bouddhistes. Il est arrivé néanmoins que ces mêmes communautés intellectuelles aient été à l'origine d'innovations majeures lorsqu'elles ont utilisé des textes classiques pour véhiculer des commentaires dans lesquels de nouvelles interprétations pouvaient être avancées. De nouveaux textes classiques du Fondateur ont pu quelquefois être " découverts », à l'exemple des sûtra Mahayana qui ont légitimé une vague nouvelle de pratique religieuse et d'activité philosophique entre 400 et 600 ans après le début du bouddhisme. Il arrive aussi que des parties obscures de textes anciens soient élevées au statut canonique, comme c'est le cas de l'adulation nouvelle qui s'attache à Mencius par le mouvement néo-confucéen aux XI ${ }^{\mathrm{e}}$ et XII ${ }^{\mathrm{e}}$ siècles. Dans le Japon des Tokugawa, une série d'innovations a pris l'aspect de l'érudition confucéenne, à 
travers la méthode qui consistait à affirmer que les textes favoris des opposants étaient des ajouts tardifs et qu'il fallait chercher la vraie doctrine dans des textes plus anciens et plus authentiques. Les membres de "l'École d'érudition ancienne» devinrent les innovateurs dans le domaine du rationalisme et de la sécularisation, très proches sous de nombreux aspects des penseurs européens des Lumières, leurs contemporains. «L'École nationale d'érudition » $d u \mathrm{XVIII}^{\mathrm{e}}$ siècle encore plus archaïsante, qui promulgua le fait que les textes primitifs japonais étaient supérieurs aux importations chinoises, donna lieu à un mouvement historiciste/romantique très élaboré, symétrique du mouvement rationaliste.

11 Il existe un espace de manœuvre même au sein d'une idéologie d'apparence rétrograde. Si le plus haut prestige revient aux textes anciens, les écoles rivales peuvent entrer en compétition pour travestir leurs innovations sous des revendications concernant la plus grande ancienneté de leurs références. Le traditionalisme des loyalistes dans la sociologie contemporaine ne présente pas un caractère aussi intense: aujourd'hui, même les marxistes et les weberiens les plus portés à la vénération de leurs héros opposeraient une dénégation consciente à la croyance en la non-perfectibilité de la vision du Fondateur. Néanmoins, une posture implicite, au niveau de l'émotion et de la pratique intellectuelle, autorise la comparaison du loyalisme de certaines traditions sociologiques avec les loyalismes passés rencontrés en Chine, en Inde et au Japon. Laissons de côté la question des conditions sociales qui sous-tendent ce parallèle, tant que nous n'aurons pas examiné plusieurs autres types de traditionalisme sociologique.

\section{Les traditions impersonnelles ou anonymes}

12 La tradition consiste ici en un lignage fait d'idées et de techniques, alors que les héros fondateurs et les textes classiques suscitent peu d'intérêt. Comme exemple on peut citer le mouvement du choix rationnel, l'analyse du rituel et du symbolisme qui a des racines durkheimiennes mais qui se développe maintenant sous l'aspect d'un lignage anonyme, les ethnométhodologues d'aujourd'hui, particulièrement ceux qui font de l'analyse de conversation, et les spécialités techniques comme l'analyse de réseaux.

13 Ces traditions présentent indubitablement des individualités remarquables. Pour le choix rationnel, on peut citer James Coleman, Gary Becker, Jon Elster et quelques autres. Mais aucun d'entre eux n'est considéré comme une figure de proue, un emblème totémique dont le nom est invoqué pour sanctifier un travail. Il n'y a ni «colemaniens» ni «beckeriens ». Dans la mesure où la théorie du choix rationnel est la réactivation ou la reconstruction d'une ancienne tradition militariste, on qualifie souvent ses hypothèses de « smithiennes ». Mais il s'agit d'un terme destiné à jeter l'opprobre, une critique venant de l'extérieur plutôt qu'un principe d'identification : ce terme n'est guère approprié pour décrire les méthodes effectivement utilisées par les membres du mouvement du choix rationnel, particulièrement les sociologues. La question de l'emblème totémique pour la tradition du choix rationnel nous aide à tracer la frontière entre deux sortes de tradition, l'anonyme et l'héroïque. L'ombre du choix rationnel s'étend au-delà de la frontière de la tradition héroïque/loyaliste lorsqu'on passe de la sociologie à l'économie orientée par la décision politique : nous rencontrons ici le loyalisme «smithien » de l'école de Chicago, dans lequel Gary Becker et Milton Friedman deviennent des "lieutenants-héros", acolytes tenant la chandelle à Adam Smith. Le degré de politisation atteint par une théorie au sein de l'arène publique est un élément qui favorise la constitution d'une 
tradition sur le mode héroïque/loyaliste. Ceci est explicable par la théorie conflictuelle de la solidarité sociale : un groupe se tourne d'autant plus vers lui-même, prête d'autant plus d'attention à ses frontières et se préoccupe d'un emblème concret d'appartenance qu'il est engagé dans un conflit ouvert, comme ceux qui se développent dans l'arène politique ${ }^{3}$. En tant que mouvement universitaire, d'un autre côté, la théorie du choix rationnel exemplifie la tradition anonyme et impersonnelle. Ceci renvoie en partie au fait que ses racines ne remontent pas à un fondateur singulier. Depuis 1970 l'économie est devenue une alliée du choix rationnel, et pour certains de ses membres une caractéristique définitionnelle, à tel point que cette théorie est quelquefois considérée comme l'expression pure et simple de l'impérialisme des méthodes économiques en sociologie. Néanmoins si les sociologues du choix rationnel devaient se trouver un fondateur, ils n'auraient pas besoin de se tourner vers Smith. Il existe une tradition indigène en sociologie, établie dans les années trente par Willard Waller, qui avait analysé les rencontres sexuelles des adolescents comme un marché matrimonial à l'essai. Pratiquant un élargissement par rapport à des domaines aussi spécialisés, Homans dans les années cinquante et Blau dans les années soixante ont développé ce qu'ils appellent une « théorie de l'échange » qui concentre son attention sur la recherche par les acteurs individuels de rétributions rapportées aux coûts qu'elles engendrent.

Nous pourrions rétrospectivement voir dans cette théorie une convergence avec le modèle économique, mais celle-ci ne fut pas perçue sur-le-champ. Ce ne sont pas les thèmes économiques des prix et de la production qui intéressaient Homans et Blau, mais la question de savoir comment les institutions sociales pouvaient se développer à partir d'incitations individuelles. Ils contestaient ainsi l'analyse fonctionnaliste orientée du haut vers le bas et centrée sur l'analyse des valeurs et de la culture. Le résultat fut de soulever le problème de l'articulation du micro et du macro, laquelle devait devenir un enjeu croissant dans la lutte entre les traditions sociologiques rivales au cours des années soixante-dix et quatre-vingts. En outre, à la différence de l'économie, la «théorie de l'échange » n'a pas fait entrer dans son champ de vision des marchés réunissant un grand nombre de participants sur une large échelle et leurs équilibres, mais une série d'arrangements locaux s'apparentant à des trocs entre quelques acteurs sociaux. De cette différence d'approche est issu un des points clés de la relation entre la nouvelle sociologie de l'échange et l'économie traditionnelle : le degré auquel la concurrence sur le marché et la formation des prix sont limitées par des réseaux sociaux structurés et dominés par des niches locales. Le mouvement du choix rationnel ne peut être réduit à la manifestation d'un impérialisme économique. Ayant connu des débuts indépendants de chaque côté de la frontière disciplinaire, ce mouvement a aussi donné naissance à un contreimpérialisme sociologique, associé à des noms comme ceux de Granovetter et Harrison White : l'économie conventionnelle des marchés est subsumée comme un sous-type dans le cadre plus large des échanges sociaux.

Le cas du choix rationnel suggère une autre raison susceptible de rendre compte du fait qu'une tradition prenne la forme de l'anonymat plutôt que la forme héroïque/loyaliste. La multiplicité des racines et des trajectoires internes interdit à un mouvement intellectuel de se focaliser sur un chef de lignage totémique, capable d'établir son identité par rapport aux étrangers. Qu'est-ce donc qui fait tenir ensemble un mouvement aussi divers dans une seule tradition, dans une seule communauté ? La vie intellectuelle est principalement organisée autour de controverses plutôt qu'autour de solutions. Dans ce cas, la communauté tient sa cohésion des controverses, de nature surtout techniques, qui 
se développent entre ses divers sous-groupes, plutôt que des controverses avec les étrangers. Le choix rationnel a connu son floruit en mettant au jour une série de paradoxes, qui stimulent les débats et la recherche de solutions innovantes: le dilemme du prisonnier dans la théorie des jeux, la rationalité limitée de March et Simon; le problème du passager clandestin formulé par Mancur Olson; l'énigme que constitue l'explication de la solidarité sociale en partant de l'intérêt individuel, un problème qui a conféré leur notoriété à Coleman et Hechter. La tradition du choix rationnel s'est développée autour d'une communauté consacrée à la solution de telles énigmes.

Toute tradition intellectuelle reste en vie parce qu'elle offre des objets à propos desquels ses membres peuvent travailler, publier leurs recherches, faire carrière. La fécondité des différentes espèces de tradition correspond à différentes espèces de capital intellectuel : dans cette tradition, on pourrait l'appeler " capital lié à la position des problèmes ». Du côté des traditions de type héroïque/loyaliste, le capital intellectuel qui permet aux savants de faire leur carrière réside dans l'appropriation des textes classiques, qui devient le fondement de commentaires, de supercommentaires et quelquefois d'applications à de nouveaux cas. Si les commentaires ne s'accordent pas, ou si les applications mettent à l'épreuve les limites de la tradition classique (prenons comme exemple la question de savoir si l'analyse marxienne est valide pour le système-monde sans violer la théorie de la plus-value), la situation est d'autant plus favorable à la vie de la tradition intellectuelle. Le cours actif de la tradition prend alors la forme de débats entre commentateurs rivaux, et s'exprime à travers des évaluations rivales pour déterminer la loyauté des extensions de la tradition à la recherche empirique. Peu importe de savoir qui est le vainqueur dans ces débats. L'existence d'hérésies (ou, pour être plus précis, de charges d'hérésies), loin de ruiner une tradition loyaliste, la maintient en vie, en concentrant l'attention sociale sur les emblèmes sacrés qui la constituent.

La vie d'une tradition anonyme présente un autre mode de lutte intellectuelle. La tradition du choix rationnel, nous l'avons vu, présente une multiplicité de racines, certaines en sociologie, d'autres en philosophie ou en science politique. Ce n'est pas simplement le chevauchement des appareils conceptuels utilisés dans chaque branche qui confère sa cohérence à une telle masse de matériel et en fait la base d'une communauté intellectuelle. Car il y a ailleurs dans le monde intellectuel de nombreux chevauchements qui ne sont pas autre chose que des caractéristiques d'arrière-plan dans la complexité de la vie intellectuelle, et qui ne fournissent pas de point focal particulier sur lequel l'attention puisse se fixer. L'unité sociale qui existe au sein de cette multiplicité, et qui nous autorise à parler de manière réaliste d'un mouvement du choix rationnel, est le produit du caractère fortement convergent des conflits qui le constituent. Le problème du dilemme du prisonnier est une version restreinte du problème plus large de la solidarité sociale : c'est un cousin du problème du passager clandestin. Le problème de la rationalité limitée soulève au niveau microsociologique la question des limites de l'acteur rationnel et des déterminations qui pèsent sur lui ; au niveau macro-sociologique, la question soulevée est celle de la détermination du rapport entre le marché concurrentiel de l'économiste et le réseau constitué par des niches d'échanges différenciées analysé par le sociologue. Il s'agit d'une famille de problèmes qui tissent des liens entre ceux qui échangent des arguments à leur propos et qui se trouvent réunis de ce fait en une communauté intellectuelle. Les problèmes sont techniques, et non populaires. Ils n'entrent pas aisément en résonance avec les idéologies politiques. Ils ne prennent leur sens que pour des intellectuels spécialisés qui peuvent les reconnaitre comme des 
paradoxes dans les termes définis par les hypothèses de base de leur tradition. Quelque degré de radicalité qu'une solution à un paradoxe de la théorie du choix rationnel puisse présenter, elle reste à l'intérieur de la tradition dans la mesure où une argumentation trouve son audience uniquement parmi les membres de la tradition. L'unification de la tradition à partir des frontières sociales tacites qu'impliquent les problèmes considérés constitue une alternative à l'unification d'une tradition par la référence à des fondateurs totémiques.

$\mathrm{Au}$ lieu de s'orienter de manière rétrospective vers ceux qui sont à son origine, une tradition anonyme est tendue vers l'avenir. Il ne serait pas exact de dire pour autant que la tradition anonyme est cumulative et qu'elle engrange progressivement des résultats alors que la tradition héroïque/loyaliste est non cumulative et stagnante. Cela reviendrait à confondre les symboles d'appartenance d'une communauté intellectuelle avec sa production effective au cours du temps. Les traditions marxienne et weberienne, bien qu'elles aient été orientées vers les vérités indestructibles proférées par leurs fondateurs héroïques, ont connu de nombreux développements. Un observateur extérieur serait en mesure d'établir le caractère cumulatif de diverses sortes de connaissance (à propos des économies mondes, des ruptures révolutionnaires ou des conflits de pouvoir dans les organisations par exemple), même si en son propre sein une telle tradition ne voit qu'obstination ou hérésie. D'un autre côté, on constate que le progrès de la théorie du choix rationnel repose largement sur l'approfondissement de la compréhension des paradoxes et des problèmes complexes qui affectent ses concepts centraux. Néanmoins le contraste persiste entre le profil social de ces deux types de traditions : l'une est orientée vers le passé et détourne son attention de sa propre histoire et de ses réalisations ; l'autre est programmatiquement tournée vers le futur.

Lorsque je suggère que la tradition durkheimienne est devenue une tradition anonyme, j'introduis un degré de plus dans la complexité: dans certaines circonstances, une tradition héroïque/loyaliste peut-elle se muer en une tradition anonyme? Au tournant du siècle il existait une école durkheimienne à tous les sens du terme: un réseau solidement organisé, poursuivant avec succès une politique de conquête de positions universitaires et contrôlant son propre programme de publications. Durkheim créa délibérément, et avec succès, les textes classiques de la science qu'il avait constituée. La reconnaissance publique suivit: vers 1920 les travaux de Durkheim étaient cités à la Chambre des Députés. Mais la lignée organisée s'est interrompue. On dit souvent que l'école fut détruite par la Première Guerre mondiale, au cours de laquelle un bon nombre de jeunes durkheimiens perdirent la vie. L'explication est superficielle. Dans d'autres cas une tradition a pu être construite à partir d'un lignage plutôt étroit fondé sur la transmission personnelle. Dans le cas du durkheimisme plusieurs membres survécurent, et particulièrement Mauss, qui avait été le lieutenant de Durkheim : il devint le maillon principal de la chaîne qui transmit la tradition aux générations suivantes. Le durkheimisme fleurit en fait jusqu'aux années vingt, mais dans les années trente sa réputation s'était retournée contre lui : on ne le considérait plus comme une théorie progressiste, mais comme une théorie dogmatique, voire même réactionnaire ${ }^{4}$. Son succès politique dans l'arène populaire conduisit aux dangers inévitables que recèle l'entrée dans l'univers fluctuant des conflits de pouvoir politique. Les Durkheimiens, avec leur souci de la solidarité sociale, devinrent anathème pour la gauche qui avait oublié leur ancienne relation avec les dreyfusards et le socialisme ${ }^{5}$. Nous voyons encore ici à l'œuvre un modèle de tradition héroïque/loyaliste. L'ancrage dans la politique entraîne une 
orientation d'allure rétrograde envers le héros fondateur, et une fermeture défensive des frontières. Comme c'est le cas pour le marxisme, qui a subi une éclipse chez les intellectuels occidentaux après l'effondrement de l'URSS, nous constatons que les traditions intellectuelles qui vivent de la politique meurent aussi de la politique.

Néanmoins la tradition durkheimienne ne mourut pas. Les idées qu'elle porte ont persisté et ont connu une expansion au point qu'on peut décrire une bonne partie des sciences sociales - c'est-à-dire un des territoires majeurs de la vie intellectuelle depuis 1940 comme étant durkheimienne. La tradition est simplement devenue anonyme. Coupées de la référence prééminente à son fondateur, mais fidèles aux modes d'analyse durkheimiens, plusieurs branches ont fleuri, qui ne manquent pas de lignes de transmission intergénérationnelle. On peut schématiquement distinguer :

1. une branche macro-sociologique, faisant fond sur le fonctionnalisme durkheimien et sur l'évolution de systèmes différenciés. C'est la lignée de Parsons et de ses successeurs, jusqu'à ses derniers élèves comme Luhmann et Alexander.

23 2. une branche micro-sociologique, intéressée au premier chef par les rituels et par la production locale de solidarité de groupe et de ses symboles. Ici la lignée s'est développée à partir de contacts entre Durkheim, Mauss et Radcliffe-Brown, par l'intermédiaire d'anthropologues sociaux britanniques, jusqu'à Lloyd Warner (qui étudia le rituel et le symbolisme des classes sociales modernes) et à Mary Douglas (qui a généralisé l'analyse des barrières tribales contre la souillure en une théorie de la "grille et du groupe ", dimensions des barrières symboliques présentes dans la structure statutaire de toute société). Erving Goffman, élève de Warner, fit de cette branche de la lignée durkheimienne un courant à la fois anonyme et activement micro-sociologique, en forgeant la notion de "rite d'interaction » (interaction ritual) pour décrire les solidarités éphémères que suscitent les rencontres sociales et en analysant la mise en scène des sois multiples comme un micro-symbolisme de l'ordre interactionnel lui-même. L'école goffmanienne est souvent mésinterprétée comme une branche de l'interactionnisme symbolique, du fait que Goffman fut associé dans les années cinquante au département de sociologie de l'université de Chicago. En fait Goffman se montra toujours vivement critique à l'égard de Blumer et des interactionnistes symboliques. Alors que ceux-ci mettaient l'accent sur le caractère négocié des situations et sur leur fluidité, Goffman défendit le caractère contraignant des structures supra-individuelles par rapport aux situations, ce qu'Anne Rawls appelle "l'ordre de l'interaction sui generis». Avec l'émergence de la sociologie des émotions (Scheff, Kemper), des chaînes de rites d'interaction et du ritualisme social en général, la tradition micro-sociologique durkheimienne en est maintenant à la quatrième génération.

24 3. une tradition structuraliste, préoccupée par les systèmes symboliques comme constituants de l'ordre social en profondeur. Cette branche a attiré l'attention sur elle avec Lévi-Strauss, qui a construit sa théorie à partir du travail de Mauss. Les disciples de Saussure (qui avait lui-même à l'origine des sympathies durkheimiennes) ont constitué une autre connexion, lorsque la linguistique structurale s'est élargie à un programme d'ensemble pour les sciences sociales autour des années soixante.

Nous voyons ici une raison supplémentaire de l'anonymisation d'une tradition héroïque/ loyaliste. Le processus de constitution de branches met l'accent sur les différences entre les lignages et obscurcit leur origine commune. Les conflits entre lignages sont au centre de l'attention. Les disciples de Goffman devinrent des micro-sociologues militants en attaquant les fonctionnalistes parsoniens ${ }^{6}$. Bourdieu offre un autre exemple de relation: 
héritier des débats sur le structuralisme, il est entré dans le champ en attaquant le structuralisme de Lévi-Strauss. Comme les idées sont structurées par leurs oppositions, l' habitus de Bourdieu a un air de famille avec la structure profonde sous-jacente aux systèmes symboliques et aux institutions sociales, qui était au centre de la conceptualisation de Lévi-Strauss. Dans une perspective longue, les écoles de Bourdieu et de Lévi-Strauss ont développé la tradition inaugurée par Durkheim et Mauss en 1903 dans leur essai sur les formes primitives de classification. Bourdieu illustre une autre caractéristique de la tradition anonyme: il mélange les ingrédients de différentes traditions. Le durkheimisme est mixé avec des aspects du marxisme (qui constitue la culture commune dans la vie intellectuelle française des années soixante et soixante-dix) et avec le weberianisme (héritage de son maître Aron, qui introduisit Weber en France en 1935 avec la Sociologie allemande contemporaine ${ }^{7}$ ). De tels croisements de traditions réduisent nécessairement l'élément de type hérö̈que/loyaliste : on ne peut pas vénérer des maîtres rivaux avec le même degré d'intensité que celui qui n'a qu'une vénération exclusive. Et la combinaison de plusieurs espèces de capital culturel est en elle-même une source d'innovation, en ce qu'elle fait avancer la tradition vers de nouvelles positions.

Les ethnométhodologues fournissent une illustration supplémentaire du processus d'anonymisation qui peut affecter un mouvement sociologique au cours du temps. Cette affirmation peut sembler ironique, dans la mesure où Garfinkel, le fondateur, a la réputation d'être une des personnalités les plus hautes en couleur de la sociologie et où, pendant les premières décennies, le réseau des ethnométhodologues était si compact qu'on l'assimilait à un culte. Les jeunes ethnométhodologues qui vivaient à Berkeley dans les années soixante étaient l'objet de nombreuses rumeurs: on leur prêtait ainsi l'habitude de lire les manuscrits non publiés de Garfinkel à la lueur d'une chandelle. Ils étaient quelquefois qualifiés par des personnes extérieures au mouvement de "sociologues à tête acide", ce qui laissait entendre que la vision qu'ils avaient de la construction de la réalité quotidienne était influencée par l'usage de LSD. En fait, le réseau était à la fois dense et exclusif: il était organisé sur la base d'invitations personnelles à des rencontres purement privées. L'hostilité à l'égard des étrangers était à la fois structurale et doctrinale. L'ethnométhodologie se pensait en contraste avec la "sociologie» en général, celle-ci étant décrite comme une forme parmi d'autres de réalisation par des agents sociaux, qui confondait un objet d'étude et une ressource et ne prenait pas en compte l'analyse de ses propres techniques, prises comme allant de soi, de construction du sens du social. L'hostilité qui en résulta de la part des sociologues contribua à durcir encore les frontières et à intensifier le sentiment de solidarité dans le cercle des fidèles.

Une telle situation constitue le fondement d'une tradition héroïque/loyaliste. Nous trouvons d'ailleurs des éléments de cette espèce de loyalisme au début du mouvement. Le texte de Garfinkel fut traité comme un classique. On fit des recherches en amont pour retrouver des textes plus précoces, particulièrement ceux de Schutz, de Gurwitsch, Die Krisis der europäischen Wissenscbaften de Husserl. Pourtant en l'espace de quinze ou vingt ans l'ethnométhodologie évolua vers un mouvement relativement anonyme, avec un intérêt moindre pour les textes fondateurs que pour ses problèmes en cours et ses découvertes empiriques.

28 Cette transformation s'explique en partie par l'engagement de l'ethnométhodologie dans la recherche. "Ethnométhodologie » signifie «ethno-méthodologie », c'est-à-dire l'ethnographie des méthodes par lesquelles les agents sociaux donnent sens à la réalité 
ordinaire. Garfinkel lui-même a été plus un chercheur qu'un auteur. Son unique livre, Studies in Ethnomethodology, est un recueil de rapports de recherche rassemblés pour la publication par Cicourel, qui était alors le lieutenant de Garfinkel. Garfinkel, élève de Parsons et de Schutz, aurait pu se contenter de faire des synthèses théoriques, en prenant l'analyse de Schutz comme point de départ pour une critique et une alternative à Parsons sur la question des éléments constituants du social. Mais Garfinkel avait commencé ses travaux dans les années cinquante, une ère qui avait vu la sociologie fondée aux EtatsUnis sur la "science du comportement »: ses premières recherches, dont certaines avaient été commanditées par le Bureau de la recherche scientifique de l'US Air Force, élargissaient le paradigme conventionnel de l'expérimentation en psychologie sociale. Il s'agissait, pour ainsi dire, d'ouvrir une brèche dans les murs du laboratoire et de permettre à l'expérimentateur de faire ses frasques dans le monde ordinaire.

Garfinkel assigna à l'ethnométhodologie un programme qui consistait à cartographier les procédures de raisonnement tacite dans la vie sociale ordinaire, à l'aide de formes ingénieuses d'expérimentation (comme le dérangement et d'autres formes de perturbation conceptuelle). Ce programme eut des ramifications dans différentes niches. Certains poursuivirent l'ethnométhodologie "classique" de la vie quotidienne, particulièrement dans les places fortes californiennes comme Santa Barbara, Los Angeles ou San Diego. Le mode loyaliste y resta le plus fort.

D'un autre côté, «l'école des études de laboratoire » en sociologie des sciences constitua une des branches les plus prospères et les plus dynamiques de ce mouvement. Tout commença par la visite de jeunes chercheurs européens comme Latour, Woolgar, et Knorr en Californie du Sud dans les années soixante-dix : ils entrèrent en contact avec Cicourel, Garfinkel et d'autres membres du réseau ethnométhodologique, et appliquèrent les méthodes de l'ethnographie radicale à la construction locale du savoir dans les laboratoires scientifiques. Le résultat fut de rendre l'ethnométhodologie cosmopolite. Le centre de l'activité s'éloigna des réseaux denses de la Côte Ouest et se déporta principalement vers la Grande-Bretagne, où un réseau plus large de chercheurs créa plusieurs centres et une série de colloques dynamiques. Le réseau élargi acquit une identité séparée sous le nouveau label de Sociology of Scientific Knowledge (SSK). Le radicalisme épistémologique de l'ethnométhodologie trouva dans ce secteur des conditions de réception plus favorables que dans la sociologie générale, en partie parce que celui-ci avait des racines philosophiques (particulièrement la nouvelle génération husserlienne). L'innovation clé de Garfinkel consista à exporter les questions épistémologiques de l'abstraction vers la recherche de terrain effectuée dans des cadres naturels. Les sociologues radicaux de la science captèrent l'attention (souvent hostile, à coup sûr) des philosophes de la science, à la différence de la sociologie des sciences pratiquée par la génération antérieure qui s'était cantonnée à l'analyse des institutions et avait évité d'aborder les questions épistémologiques. Ce mélange disciplinaire dans l'espace d'attention eut pour effet d'arracher l'ethnométhodologie au mode hérö̈que/ loyaliste. Le mouvement du constructivisme social, qui domine la sociologie des sciences depuis 1980, a perdu conscience de la nature ethnométhodologique de ses racines. L'ethnographie radicale de la vie de laboratoire s'est mélangée à divers mouvements proches (comme l'école d'Edimbourg de Bloor et Barnes, qui a bâti son projet de sociologie de la connaissance à partir d'autres voies, notamment Durkheim, Wîttgenstein et Kuhn). Nous constatons à nouveau que l'intrication des réseaux, dont la cohérence 
tient au partage d'un ensemble commun d'arguments ésotériques, transforme une tradition héroïque/loyaliste en tradition anonyme.

31 On peut dire la même chose d'une autre ramification très prospère de l'ethnométhodologie, l'analyse de conversation (celle-ci a pris à ce point ses distances avec l'ethnométhodologie qu'elle a son propre surnom «CA»). Le point de départ de l'analyse de conversation est au cœur du réseau ethnométhodologique, avec les premiers disciples de Garfinkel, Sacks et Schegloff. Le caractère distinctif de ce type d'analyse s'affirma pourtant lors de l'expansion de l'école ethnométhodologique à la deuxième génération (Heritage, Boden, Clayman, et Schegloff qui poursuivait son travail). Si l'accent continue d'être mis sur le radicalisme ethnographique et la production locale de tous les phénomènes sociaux, l'analyse de conversation a développé un souci de formalisation et la recherche de lois universelles. Celle-ci est de plus en plus étrangère à l'esprit antipositiviste de l'ethnométhodologie orthodoxe, particulièrement lorsqu'il s'exprime dans ses préoccupations classiques à propos du caractère incontournable du savoir local tacite ou de la régression infinie qu'entraîne la réflexivité. Le déplacement de l'ethnométhodologie vers l'analyse de conversation peut être attribué au développement d'une nouvelle identité de base produite par une pratique de recherche spécifique, à savoir la méthode qui consiste à analyser des enregistrements de conversations naturelles, avec le système de notation formelle inventé par Sacks, Jefferson et Schegloff. La méthode conduit tout naturellement à l'accumulation de résultats de recherche sous une forme abstraite et partant généralisable. L'analyse de conversation s'affranchit des textes classiques de l'ethnométhodologie et développe ses propres théories. L'éloignement par rapport au mode textuel est repérable dans le changement de position de l'œuvre d'Harvey Sacks. Les conférences de Sacks, enregistrées par ses étudiants au début des années soixante-dix, avant sa mort dans un accident de voiture, furent pendant des années un objet d'attention s'apparentant à un culte de la part de ceux qui y avaient accès. Leur publication en $1992^{8}$ ne suscita guère d'attention, même dans l'univers de l'analyse de conversation : le mouvement avait évolué vers le mode anonyme.

Les traditions techniques en sociologie sont toutes transmises sur le mode anonyme, même si certains traits de leur organisation semblent favoriser le loyalisme héroïque. On constate que le réseau de recherche est constitué par une communauté très soudée pourvue d'une conscience militante dirigée contre les étrangers. Depuis les années soixante-dix les chercheurs du réseau ont tenu les "Colloques de la Sun Belt» et entretenu leurs propres canaux de communication. Le mouvement a développé une idéologie de sa propre pureté, entretenue par des militants qui opposent le mélange d'analyse structurale de réseau avec des éléments étrangers de psychologie de la culture ou de psychologie individuelle. Ces militants croient qu'ils ont pour mission de sauver la sociologie de l'impérialisme de l'analyse de réseau. Ces idéologies sont en affinité avec la structure dense et fermée de cette communauté de recherche. Bien qu'elle réunisse toutes les conditions de la haute densité morale au sens durkheimien, cette communauté n'a jamais rendu hommage à un fondateur totémique (bien qu'on connaisse évidemment des noms de pionniers), pas plus qu'elle ne défend des textes classiques. La comparaison nous permet de voir que la source du loyalisme héroïque ne résulte pas seulement d'une forme d'organisation qui s'apparente au culte, mais de la différence entre les modes de solidarité qu'induisent d'un côté le centrage sur des techniques, et d'un autre côté le centrage sur des textes. 

caractère "hard» ou "soft» des méthodes ou des schèmes d'analyse. Du côté de la tradition anonyme, nous trouvons à la fois des approches hautement formalisées, s'appuyant sur les mathématiques (l'analyse de réseau) aussi bien que de l'ethnographie qualitative (la sociologie du savoir scientifique comme surgeon de l'ethnométhodologie, la micro-sociologie durkheimienne dérivant de Goffman). La différence ne repose pas non plus sur la distinction entre des intellectuels qui font de la recherche empirique et d'autres qui se contentent d'étudier les écrits de théoriciens antérieurs. La recherche empirique peut constituer aussi un mode de théorisation, comme on le voit dans les travaux de Marx, d'Engels et de Weber, même si ceux-ci sont aujourd'hui surtout interprétés comme des travaux théoriques. La recherche historique est aussi une rencontre avec le monde de l'empirie (et de fait la seule manière de saisir à une échelle significative une dynamique macro-sociologique). La recherche historique est aisément assimilée au mode héroïque/loyaliste. L'explication de ce point de vue semble résider dans le fait que ce type de recherche s'exerce généralement sur des textes. L'activité sociale pratique qui consiste à construire une argumentation à partir de textes diffère des pratiques de recherche qui passent par l'observation ethnographique ou par l'usage d'instruments (qu'il s'agisse de magnétophones, d'ordinateurs ou d'opérations statistiques). Sous certaines conditions, il devient possible de vénérer le texte idéal, en le transformant en emblème totémique de l'appartenance au groupe. Ceci apparaît particulièrement bien dans le cas de l'érudit loyaliste qui écrit des commentaires sur les textes de Weber ou de Mead. Mais le spécialiste de sociologie historique, qui peut être engagé dans une réflexion substantive (sur l'émergence du capitalisme, sur la bureaucratie, etc.), peut néanmoins tomber dans la célébration d'un texte (celui-ci pouvant être mythique) comme un classique : c'est l'image du maitre-texte qui permet d'interpréter tous les textes.

Nous avons vu que plusieurs conditions organisationnelles de la vie intellectuelle conduisent à des modes de tradition spécifiques, loyalistes ou anonymes: une densité morale forte - au sens durkheimien - de la communauté intellectuelle elle-même, une condition de fond favorable au loyalisme à l'égard du héros mais qui ne parvient pas à s'exercer pleinement; la participation à l'arène politique du conflit, qui durcit les frontières idéologiques; l'intrication entre plusieurs réseaux intellectuels, qui affaiblit généralement le centrage de l'attention et élimine l'identité collective consciente, sauf lorsqu'un réseau d'origines multiples se centre autour d'un lignage spécifique constitué d'énigmes ésotériques; et enfin le centrage de la pratique intellectuelle sur des techniques textuelles ou non textuelles. Aucun de ces facteurs n'est suffisant pour déterminer le mode de tradition. Les préoccupations textuelles ne conduisent pas nécessairement à des traditions : elles peuvent conduire à des antitraditions, comme nous le voyons dans le cas des postmodernes et des historicistes. Avant d'en venir à ce point, nous devons aborder un autre type de tradition.

\section{Les traditions avortées}

Il serait trop simple de donner l'impression que le champ de la sociologie est simplement divisé en deux traditions. La plus haute forme de réussite dans le monde intellectuel est de fonder une tradition. Tous n'y parviennent pas. La vie intellectuelle est une compétition pour placer son travail au centre de l'attention. L'espace d'attention propre 
au public intellectuel est limité à un petit nombre d'écoles opposées dans chaque spécialité. Les milliers de participants ne peuvent pas tous réussir à acquérir une identité propre. Si le champ est déjà riche de traditions florissantes, une stratégie raisonnable consiste à s'attacher à un lignage existant, à faire carrière dans l'espace d'attention en adoptant une identité déjà constituée. C'est ce qui rend attirant le fait d'être weberien ou parsonien, même si c'est au prix de l'oblitération de son identité personnelle. L'individu est ainsi placé sur la carte des partis reconnus dans la discipline et peut même escompter des perspectives de carrière ambitieuses, s'il a la chance de se positionner pour être interprète en chef, par exemple pour être le premier weberien de son temps. Il s'agit d'une éminence masquée, pour ainsi dire. Le rôle n'est pas dépourvu de créativité, si l'on dissimule ses innovations sous le mode du traditionalisme.

D'autres intellectuels choisissent une stratégie opposée : ils rêvent de devenir un nom propre de plein exercice. Un tel choix peut être l'effet de la jeunesse, de la naïveté et de l'éloignement par rapport aux réseaux dominants; mais il exprime aussi l'idéologie populaire de la vie intellectuelle moderne, qui exalte l'individu créatif, le rebelle qui parvient à la grandeur au prix du travail solitaire. Il existe aussi des périodes de transition, lorsqu'un champ est en cours de constitution, ou lorsque ses bases s'élargissent ou sont fortement déplacées: ces conjonctures sont favorables au développement de nouvelles écoles. De nombreux candidats au poste de fondateur apparaissent alors. Mais la loi des petits nombres qui gouverne la réussite à l'intérieur de l'espace d'attention ne permet qu'à quelques-uns d'acquérir une réputation durable. La génération du tournant du siècle - moment de l'institutionnalisation de la sociologie - a produit notre propre panthéon: Durkheim, Weber et Mead. De nombreux sociologues créatifs ont été leurs contemporains : en position éminente de leur vivant, ils n'ont pu acquérir un réseau de disciples pour transmettre la tradition à la génération suivante. On peut citer en exemple Simmel, Tarde, Sumner et bien d'autres. Nous ne pouvons pas connaitre le nombre total d'individus qui choisissent cette filière, qui rejettent l'appartenance à une tradition (qu'elle soit héroïque ou anonyme) en vue de chercher pour eux-mêmes l'éminence créative maximale. La poignée de noms dont nous nous souvenons (comme Simmel) étaient déjà des éminences de deuxième rang, généralement bien reliées aux réseaux de leur époque en sorte que leur mémoire pouvait être prise en charge par des membres du courant dominant. Au-delà de ce groupe ayant connu une demi-réussite se trouvent bien d'autres individus, savants dont les travaux n'ont jamais été publiés, ou dont les livres se sont peu vendus ou n'ont suscité guère de comptes rendus. À en juger par les données réunies par Derek Price ${ }^{9}$ sur les scientifiques, nous pouvons dire que la structure de la compétition pour l'attention dans un champ intellectuel a la forme d'une pyramide: le nombre d'intellectuels dont la réussite est modeste est beaucoup plus important que le nombre de ceux qui atteignent le sommet et qui reposent sur une base d'aspirants qui va s'élargissant en fonction du degré croissant d'obscurité.

Quand nous regardons le passé, nous ne voyons que le sommet des montagnes. Pour ce qui est de notre époque, notre expérience quotidienne nous place au milieu de la montagne: nous sommes entourés d'intellectuels aux trajectoires très diversifiées, promis à des degrés de réussite très différents dans la longue durée de la mémoire historique. Notre vision est opacifiée par les espoirs et les souhaits de voir tel ou tel rester dans la mémoire des traditions futures. Le paysage contemporain est obscurci non 
seulement par les traditions héroïque/loyaliste et anonyme, mais aussi par les traditions avortées en voie de constitution.

\section{Les antitraditions} plusieurs moyens de le faire. Le premier consiste à prendre une position historiciste, en mettant l'accent sur le caractère particulier et contextualisé de tous les événements sociaux. Dans cette perspective, on a seulement la possibilité de décrire un lieu et un moment particuliers. On ne peut faire aucune assertion théorique de portée générale; aucun objet n'a intrinsèquement plus d'importance qu'un autre. Il s'agit là d'un point de vue extrêmement égalitariste dans la communauté intellectuelle. Aucun théoricien ne s'élève au-dessus des masses. Aucune recherche n'est assez importante pour mobiliser l'attention.

Avant de se demander quelles conditions sociales peuvent donner lieu à une telle organisation du monde intellectuel, considérons une version plus militante de l'antitraditionalisme. Celle-ci prend sa forme la plus accusée dans le postmodernisme, qui déclare que toutes les traditions sont mortes et qui promulgue une méthode garantissant que la prétention de tout texte à une signification transhistorique est réduite à néant par l'examen des circonstances fugaces de sa production et de sa réception. Surgi en philosophie et en théorie littéraire, le mouvement a connu ses plus grands succès (particulièrement aux États-Unis) sous la forme d'un impérialisme des professeurs de littérature sur les autres départements universitaires. Il résulte de cette origine que l'attaque postmoderne contre les "grands récits » est particulièrement adaptée au rejet des traditions marxienne et freudienne qui ont connu une grande popularité parmi les intellectuels dans les années soixante-dix. Les ressources en capital culturel dont dispose le postmodernisme ne sont guère adaptées à la critique des traditions existantes en sociologie, lesquelles ont déjà des positions bien assurées pour traiter de la question du caractère socialement construit de la réalité, sans se limiter au niveau marxien/freudien. Le postmodernisme est antitraditionnel au niveau idéologique, mais est socialement organisé comme une tradition (il s'agit pour ainsi dire d'une tradition hyper-anonyme), puisque le noyau créatif du mouvement a été essentiellement " postmarxiste », dérivant directement de la désillusion qui frappait des marxistes renégats, lesquels ont appliqué la théorie de la marchandise au domaine de la production culturelle. C'est le marxisme dans une veine pessimiste, sans ses soubassements économiques et politiques : on n'attend plus de l'histoire une dynamique révolutionnaire, ni même une quelconque dynamique. Marx ne peut plus servir d'objet totémique, bien qu'il devienne implicitement un objet sacré en négatif, au sens où il exemplifie l'échec du "grand récit ». On confère à cet échec une signification apocalyptique, emblème de l'échec de tout récit théorique du même type. Quelles sont les conditions pour que de telles antitraditions occupent une position éminente dans l'espace intellectuel? Il existe peu de précédents dans l'histoire de la sociologie elle-même. D'ailleurs, la sociologie n'est pas un des bastions du postmodernisme: il ne constitue qu'une faction parmi des traditions mieux établies, incluant l'historicisme. On peut observer les antitraditions sur une plus large échelle dans l'histoire de la philosophie. Les périodes de surpopulation dans l'espace d'attention, lorsqu'il y a trop de factions rivales, donnent typiquement lieu à l'émergence d'une métafaction de sceptiques. Ces intellectuels prennent comme objet principal les différends 
entre leurs pairs et entre leurs prédécesseurs. Au lieu de se vouer à la recherche de la connaissance, ils orientent leur attention sur son impossibilité. Le scepticisme est une stratégie de recentrage face à l'état d'éparpillement et de chaos que présente le champ intellectuel. Ce n'est pas la substance des disputes, mais le méta-objet que constitue l'absence de résolution des disputes qui devient le centre de l'attention.

Plusieurs moments de l'histoire voient émerger le scepticisme. Chez les Grecs, l'école de Pyrrhon et de Timon apparut au $\mathrm{IV}^{\mathrm{e}}$ siècle av. JC, lors d'une prolifération d'écoles philosophiques rivales (Mégare, Cyrène, Elis, Cyzique, Abdère et l'Académie), en même temps que l'antilignage cynique, suivi par une seconde vague de fondations : le Lycée, le Portique, le Jardin d'Epicure. Les lignées sceptiques subsistèrent, au milieu d'écoles rivales, jusque vers la fin du II ${ }^{\mathrm{e}}$ siècle, au moment où l'éclectisme polycentrique des écoles hellénistiques tardives laissa la place à des formes plus simples d'affrontement entre positions chrétiennes et païennes. Dans l'Inde ancienne, la position sceptique dite de «la peste sur toutes les maisons » surgit à la génération précédant immédiatement Bouddha, quand plusieurs douzaines de factions rivales transformèrent l'espace d'attention en un boueux cloaque. Le scepticisme indien reflua quand l'émergence des lignées organisées bouddhistes et jaina recentra la vie intellectuelle autour d'un nombre maitrisable de positions clairement codifiées. Après une longue absence, le scepticisme devint prééminent dans l'Europe du XVI ${ }^{e}$ siècle quand la situation d'impasse dans laquelle se trouvaient les factions dans la philosophie universitaire (scotistes, thomistes, nominalistes) fut contournée par les humanistes antiuniversitaires qui ressuscitèrent d'anciennes factions. Le jeu fut encore compliqué par les occultistes, les mystiques et par les théologies rivales de la Réforme. Montaigne, le plus célèbre des sceptiques, émergea à partir d'un réseau en prise sur plusieurs factions, illustrant le principe général selon lequel même les antitraditions connaissent le succès parce qu'elles sont organisées comme des traditions clandestines, s'appuyant sur les ressources accumulées par des réseaux intellectuels antérieurs.

41 Ces comparaisons historiques suggèrent que le scepticisme n'est jamais la fin de l'histoire. Le scepticisme ne supplante jamais les factions rivales de son temps: il devient simplement une faction parmi d'autres, et peut même être institutionnalisé et routinisé dans les générations suivantes. Nous pouvons nous attendre à ce que les sceptiques postmodernistes, peut-être sous un autre nom, continueront dans le futur sous l'aspect d'une tradition antitraditionaliste, aussi longtemps que notre propre monde intellectuel (ce qui signifie en particulier notre organisation spécifique de la production universitaire) reste large, décentralisé et qu'il fait ainsi subir de trop fortes tensions au point focal de l'espace d'attention. La survie à long terme diminue l'impact des affirmations apocalyptiques. La proclamation de la fin des grands récits devient un récit parmi beaucoup d'autres.

Des précédents historiques montrent également que les réseaux de sceptiques peuvent conduire à de nouveaux réseaux, devenant un ingrédient pour des positions dont le message n'offre pas de caractère sceptique. Les doctrines du sceptique indien Sanjaya ont été préservées principalement parce qu'elles sont entrées dans la tradition des débats bouddhistes, au point de constituer un élément de la doctrine de l'irréalité du monde de l'expérience ordinaire, bien qu'elles contrastent fortement avec l'ontologie de ce qu'on peut trouver au-delà. Le scepticisme grec ancien, conservé dans les écoles de rhétorique, devint un élément du capital culturel à l'aide duquel Saint-Augustin construisit la philosophie qui couronnait le monde chrétien antique. Le réseau de Montaigne permit 
indirectement l'émergence de Descartes, qui se servit de techniques sceptiques pour mettre au clair les bases d'une philosophie déductive en construisant une métaphysique sur des fondements éprouvés. Sans vouloir suggérer que notre futur verra la résurrection de positions comme celles qu'ont occupées Gautama Bouddha, Saint-Augustin ou Descartes, je voudrais en conclure que le capital culturel que constitue un haut degré de réflexivité épistémologique, tel que le scepticisme l'incorpore, peut lui-même être intégré dans des traditions ultérieures qui prétendent édifier des conceptualisations positives, voire dogmatiques dans leur certitude.

43 Le futur de la sociologie appartiendra-t-il aux traditions héroïques/loyalistes, ou aux traditions anonymes, ou même aux antitraditions? (Je n'oublie pas bien sûr la prévalence numérique des traditions avortées sur toutes les autres.) J'ai déjà suggéré que les antitraditions ne peuvent pas dominer un champ intellectuel en leur nom propre. Elles présentent toujours un caractère parasitaire par rapport aux traditions qu'elles dénient. Qu'en est-il alors de l'équilibre entre le mode héroïque/loyaliste et le mode anonyme ? À partir des conditions que j'ai suggérées pour ces types d'organisation intellectuelle, il semble que toutes sont solidement institutionnalisées, ou susceptibles de réémerger dans le futur. Des réseaux intellectuels denses constituent des caractéristiques pérennes de la créativité. Leurs idéologies universitaires continueront à balancer vers le mode héroïque/ loyaliste aussi longtemps que les méthodes textuelles feront l'objet d'un usage exclusif par certains sociologues, ce qui semble probable si l'on considère les conditions de spécialisation qui prévalent dans l'université de masse. Bien que les idéologies politiques externes ne soient pas très saillantes en cette fin de siècle atone et désillusionnée, de telles conditions politiques ne sont pas éternelles: la re-politisation constituera un facteur favorable pour les traditions héroïques/loyalistes. D'un autre côté, les conditions de la vie universitaire moderne continuent de produire des chevauchements et des intrications entre les réseaux : ceci constitue un facteur de dilution de l'objet central de la loyauté, et stimule la constitution de lignages internes à partir de la résolution d'énigmes ésotériques.

L'expansion continue des techniques de recherche revêt une signification particulière pour la sociologie. La domination de méthodes d'enquête par questionnaire dans la sociologie du milieu $d u x^{e}$ siècle a tendu à installer la majorité des chercheurs en sociologie dans une communauté dispersée et dépourvue de centrage sur un objectif. Peut-être faut-il y voir une raison supplémentaire de la prise de distance croissante des traditions héroïques/loyalistes par rapport aux chercheurs empiriques, qui incarnaient l'impersonnalité bureaucratique de la vie intellectuelle, passée des traditions anonymes à l'anonymat sans tradition. L'ère de la domination des enquêtes par questionnaire cède la place à une plus grande variété de mouvements intellectuels fondés sur des techniques de recherche : le succès croissant de l'analyse de réseau, de l'analyse de conversation, de l'ethnographie de la vie quotidienne, et probablement des techniques à développer fondées sur l'utilisation de la vidéo, sur les simulations par ordinateurs et d'autres encore qu'il reste à imaginer. Pour faire bonne mesure, nous pouvons prévoir un futur peuplé de traditions sociologiques distinctives, c'est-à-dire de communautés intellectuelles organisées, qui ne sombrent pas dans l'anomie généralisée de la vie intellectuelle de masse et qui se réfèrent à des symboles anonymes plutôt qu'à des fondateurs totémiques, 
tout en maintenant les liens de loyauté personnelle entre des membres engagés dans la construction de projets communs.

\section{NOTES}

1. Sur cette controverse, voir particulièrement: The Brenner Debate: Agrarian Class Structure and Economic Development in Pre-industrial Europe, T. H. Aston \& C.H. E. Philpin, eds, Cambridge, Cambridge University Press, 1985 [N.d.T.].

2. Jeffrey Alexander est le symbole du retour à la grande théorie dans la sociologie américaine des années quatre-vingts. Il est notamment l'auteur de Theoretical Logic in Sociology, Berkeley, University of California Press, 1982, 4 vol., et de Structure and Meaning. Relinking Classical Sociology, New York, Columbia University Press, 1989 [N.d.T.].

3. Le mode d'organisation héroïque/loyaliste du marxisme est un exemple évident de ce schème. Le mode héroïque/loyaliste du freudisme constitue une variante : le mouvement n'est pas engagé dans la politique en tant que telle, mais le fait qu'il s'adresse d'abord à des audiences profanes et à des praticiens, par opposition aux savants, confère semblablement à son objet le caractère clos des idéologies de groupe conflictuelles. Le weberianisme, de son côté, n'a jamais constitué une position politique populaire, mais seulement un mouvement à l'intérieur de l'université. Le fait que le weberianisme soit devenu un loyalisme héröque à partir des années soixante est probablement le reflet $d u$ fait circonstanciel en vertu duquel les weberiens tendaient à se considérer eux-mêmes comme une alternative au marxisme, leur rival principal au sein de la macro-sociologie, lequel offrait un mixte de dimensions politiques et universitaires.

4. J. Heilbron, «Les métamorphoses du durkheimisme, 1920-1940», Revue française de sociologie, 26,1985, p. 220-237.

5. M. Fournier dans son livre, Marcel Mauss, Paris, Fayard, 1994, fournit des informations très importantes sur la génération universitaire influencée par les Durkheimiens dans les années trente, alors même que la réputation plus large de l'école s'effondrait. À la place de l'école héroïque/loyaliste émergea un réseau composé principalement d'ethnologues.

6. L'auteur de cet article, qui fut à la fois un élève de Parsons et de Goffman, infère que les liens personnels qui existent entre les deux parties de tels conflits n'empêchent pas un individu de prendre parti, mais donnent au contraire au débat une intensité émotionnelle sur laquelle il centre sa propre carrière.

7. R. Aron était un cousin de Mauss et fut associé aux durkheimiens au début de sa carrière (Fournier, op. cit., p. 641-642). La vie intellectuelle est structurée par des conflits et l'influence d'Aron est issue de sa participation à plusieurs réseaux différents, y compris le cercle de Sartre, auquel il fournit quelques-unes de ses connexions originales avec la philosophie allemande.

8. H. Sacks, Lectures on Conversation, éd. par Gail Jefferson, Oxford, Blackwell, 1992.

9. Derek de Solla Price est l'auteur d'études sur la morphologie et les modes de fonctionnement des communautés scientifiques analysées notamment à travers les réseaux de publications. Voir notamment «Networks of Scientific Papers", Science, 149, 1967, p. 510-515, et surtout Little Science, Big Science and Beyond, New York, Columbia University Press, 1986, nouvelle édition posthume d'un livre dont la première édition est de 1963 [N.d.T.]. 


\section{RÉSUMÉS}

Bien que les sociologues se présentent ordinairement comme ceux qui dévoilent les traditions, leur mode d'organisation intellectuelle peut être décrit en termes de tradition. On peut distinguer en sociologie quatre types de tradition, types qui ne sont pas construits par rapport à des contenus, mais à partir des relations que les groupes entretiennent avec leur propre symbolisme. On peut opposer très nettement les deux premiers types de tradition : le premier (tradition loyaliste ou orientée par des héros) est centré autour d'un fondateur dont la théorie conserve intégralement dans l'histoire son pouvoir explicatif : c'est le cas des grandes théories attachées au nom propre des pères fondateurs. Le second type (tradition impersonnelle ou anonyme) consiste en une série d'idées et de techniques, alors que les héros fondateurs et les textes classiques suscitent peu d'intérêt. Un bon exemple de ce type est fourni par la théorie du choix rationnel. Au lieu de s'orienter vers sa propre origine, comme la tradition loyaliste, une tradition anonyme est tendue vers l'avenir. On ne peut pas dire pour autant que la tradition anonyme est cumulative alors que la tradition héroïque/loyaliste est à la fois non cumulative et stérile. Cela reviendrait à confondre les symboles d'appartenance caractéristiques d'une communauté intellectuelle avec sa production effective. Il existe des formes d'innovation spécifique à chaque tradition. De plus, on constate des passages d'un mode de tradition à un autre : c'est le cas en particulier de la tradition durkheimienne. Le troisième type de tradition, numériquement le plus nombreux, est constitué par les tentatives avortées de création d'une tradition. Enfin, le quatrième type est celui de l'anti-tradition, dont le postmodernisme constitue aujourd'hui un bon exemple. Celle-ci présente toujours un caractère parasitaire par rapport aux autres traditions. L'analyse permet de conclure de manière conjecturale: les traditions sociologiques du futur se référeront probablement à des symboles anonymes plutôt qu'à des héros fondateurs, tout en maintenant les liens de loyauté personnelle entre leurs membres.

Although sociologists usually present themselves as those who unveil traditions, their mode of intellectual organization can be described in terms of tradition. Four types of tradition can be distinguished in sociology, types which are not constructed in relation to content but on the basis of relationships which groups maintain with their own symbolism. One can very clearly oppose the first two types of traditions, the first (loyalist tradition or oriented by heroes) is centered around a founder whose theory preserves its explanatory power entirely in the course of history: this is the case of big theories attached to proper names of founding fathers. The second type (impersonal or anonymous tradition) consists of a series of ideas and techniques while the founding heroes and classic texts excite little interest. A good example of this type is provided by the theory of rational choice. Instead of orienting itself towards its own origin, like the loyalist tradition, an anonymous tradition is directed towards the future. One cannot for all that say that the anonymous tradition is cumulative while the loyalist or heroic tradition is at the same time non cumulative and sterile. This would amount to confusing the symbols of adherence characteristic of an intellectual community with its actual production. There are forms of innovation specific to each tradition. Moreover, the transition from one mode of tradition to another is established: this is particularly the case with the durkheimian tradition. The third type of tradition, numerically the most profuse, is constituted by abortive attempts to create a tradition. Finally, the fourth type is that of antitradition, of which post modernism today 
constitutes a good example. The latter always presents a parasitical character as compared to other traditions. The analysis allows a conjectural kind of conclusion: future sociological traditions will probably refer to anonymous symbols rather than founding heroes, while maintaining links of personal loyalty between their members. 